Revista de la red interuniversitaria de estudios sobre las literaturas rioplatenses contemporáneas en Francia

$14 \mid 2016$

Levrero

\title{
Las personalidades múltiples de Jorge Mario Varlotta Levrero
}

\section{Diego Vecchio}

\section{OpenEdition}

\section{Journals}

Edición electrónica

URL: http://journals.openedition.org/lirico/2301

DOI: 10.4000/lirico.2301

ISSN: 2262-8339

Editor

Réseau interuniversitaire d'étude des littératures contemporaines du Río de la Plata

\section{Referencia electrónica}

Diego Vecchio, «Las personalidades múltiples de Jorge Mario Varlotta Levrero », Cuadernos LIRICO [En línea], 14 | 2016, Puesto en línea el 07 junio 2016, consultado el 02 mayo 2019. URL : http:// journals.openedition.org/lirico/2301; DOI : 10.4000/lirico.2301

Este documento fue generado automáticamente el 2 mayo 2019.

\section{(c) $(1) \odot \ominus$}

Cuadernos LIRICO está distribuido bajo una Licencia Creative Commons Atribución-NoComercialSinDerivar 4.0 Internacional. 


\title{
Las personalidades múltiples de Jorge Mario Varlotta Levrero
}

\author{
Diego Vecchio
}

\section{1- El portero y el otro}

1 El año 1992 marca un corte en la obra de Jorge Mario Varlotta Levrero.

2 En 1992, Levrero publica, en Montevideo, en la editorial Arca, El portero y el otro. Con "Apuntes bonaerenses" y "Diario de un canalla", Levrero inventa un híbrido de diario íntimo, reflexiones ecolo-cósmico-religiosas y dibujos animados, inaugurando, parece ser, una nueva etapa que se prolonga en $E l$ discurso vacío y culmina triunfante en La novela luminosa.

3 Al igual que ciertos animales unicelulares, Levrero se escindiría en dos : un Levrero 1 y un Levrero 2. Entre ambos Levreros, existiría una relación, no exenta de darwinismo positivo o negativo. Para algunos lectores, Levrero 2 es una especie que supera a Levrero 1, del mismo modo que Homo sapiens es una versión que aventaja a Australopithecus. Para otros, por el contrario, representaría una suerte de mutación degenerativa, bajo los efectos de las toxinas narcisistas de la literatura contemporánea.

Como toda clasificación, esta fisión binaria hizo posible ciertas lecturas, como así también nos escamoteó otras, impidiendo ver aquello que se articula y se reconfigura de Levrero 1 a Levrero 2, y sobre todo, aquello que hizo posible que ahí hubiera una obra atribuida a Mario Levrero.

De hecho, más que sucesivos, ambos Levreros son simultáneos. Levrero 1 y Levrero 2 coexisten en un mismo libro de relatos, que se cierra, por otro lado, con una entrevista a Mario Levrero por Mario Levrero. Mientras Levrero 2 publica en $1996 \mathrm{El}$ discurso vacío, Levrero 1 publica, también en 1996, para no ser menos, El alma de Gardel. Mientras Levrero 2 está absorbido por La novela luminosa, Levrero 1 escribe Los carros de fuego.

6 Etc.

Cuadernos LIRICO, 14 | 2016 


\section{2- Nick Carter}

En 1992, Levrero publica, también, en la misma editorial, Nick Carter se divierte, mientras el lector es asesinado y yo agonizo : folletín. Con este título kilométrico, que los diseñadores de tapas no saben muy bien cómo presentar, Nick Carter marca un giro, menos ruidoso que el primero, aunque seguramente mucho más determinante. En realidad, se trata de una reedición. El libro había sido publicado en 1974, por Jorge Varlotta, en Buenos Aires. En 1992, el mismo libro es publicado, en Montevideo, por Mario Levrero. Este simple cambio de nombre hace posible el viraje que se produce hacia el final de El portero y el otro. La anexión que hace Mario Levrero del libro de Jorge Varlotta es una condición de posibilidad de la irrupción de un Levrero 1 y un Levrero 2 (y, si se quiere, de un Levrero 3 , $405)$.

\section{3- La confusión de nombres}

Quien recorra las páginas de Un silencio menos, la compilación de entrevistas que hizo Elvio Gandolfo, advertirá cierta vacilación en los entrevistadores para designar al autor entrevistado.

Elvio Gandolfo escribe (15 de abril de 1979) : "Nació en Montevideo en 1940, y a partir de 1966 desplegó, como Mario Levrero o con otros seudónimos, diversas formas de expresar su personalidad"1.

Rafael Courtoisie (2 de septiembre de 1982) : "Sucede que inevitablemente, existen casos atípicos, difíciles de encasillar y a veces, difíciles de comprender. Hasta que llega la hora de conocerlos. Uno de estos casos se llama Mario Levrero. Su nombre verdadero es Jorge Varlotta"'.

11 Eduardo Berti y Jorge Warley (diciembre de 1986) : “La ventana estalló en pedazos. Nick Carter irrumpió en la habitación con un atemorizante revólver en la mano :

- Ando en busca de Jorge Varlotta, el montevideano.

Desde la sombra, una voz confusa respondió : 'Has errado la pista. Aquí vive Mario Levrero, porteño desde hace un año'"’3.

Más adelante, los entrevistadores aclaran: "Quien habla es un escritor uruguayo bautizado Mario Jorge Levrero Varlotta, pero que usa indiscriminadamente los nombres de Jorge Varlotta o de Mario Levrero, como intentando desconcertar a todo aquel que quiera seguir palmo a palmo su obra"4.

Cristina Siscar (mayo de 1987) : "Jorge Mario Varlotta Levrero nació en Montevideo en 1940 y vive en Buenos Aires desde hace dos años"

Pregunta : ¿Varlotta es Levrero o Levrero es Varlotta ?” Respuesta: "Ahora me estoy unificando bastante : Jorge Mario Levrero Varlotta". (Entrevista realizada el 17 diciembre de 1988 por Luis Pereira)"6.

Estos ejemplos bastan para poner en evidencia un problema que plantea nuestro autor (o mejor dicho, nuestros autores) : la confusión del nombres. ¿Mario Levrero es pseudónimo de Jorge Varlotta? O al revés : ¿Jorge Varlotta es un pseudónimo de Mario Levrero ? ¿Se trata de pseudónimos? ¿Cuál es el verdadero nombre de este autor? ¿Mario Jorge Levrero Varlotta? ¿Jorge Mario Varlotta Levrero ? ¿Jorge Mario Levrero Varlotta? 


\section{4- El primer autor bífido}

16

Por cierto, Mario Levrero no es un pseudónimo de Jorge Varlotta; como así tampoco Jorge Varlotta es un pseudónimo de Mario Levrero. Un pseudónimo es un nombre inventado por un autor, diferente de su nombre civil, para ocultar su identidad o para disociar vida cotidiana y actividades literarias. Lewis Carroll es el pseudónimo de Charles Lutwidge Dodgson. Lautréamont es el pseudónimo de Isidore Lucien Ducasse. Por cierto, ni Jorge Varlotta ni Mario Levrero corresponden a esta categoría.

A diferencia de la mayor parte de los autores que firman sus libros con un mismo nombre, como para garantizar la identificación de su producción y acumular de esta manera, por así decir, capital simbólico, Jorge Mario Varlotta Levrero lleva a cabo una práctica singular que consiste en inventar, a partir de su nombre civil, dos nombres de autor. Con su primer nombre de pila y apellido paterno, fabrica un Jorge Varlotta y con su segundo nombre y el apellido materno, un Mario Levrero. Gracias a esta operación, Jorge Mario Varlotta Levrero se convierte en el primer autor bífido de la historia, desdoblando aquello que, en principio, suele ser Uno y Único : el nombre propio.

\section{5- Lógica}

A diferencia de los nombres comunes, que se refieren a una clase de objetos, los nombres propios se refieren a un objeto particular. Aristóteles se refiere a un filósofo griego. Además de referir, los nombres propios también describen. Aristóteles está asociado a cierto número de características, tales como "hijo de Nicómaco", "discípulo de Platón", "preceptor de Alejandro", "fundador del Liceo de Atenas". A estas dos funciones, características de los nombres propios en general, habría que agregar una tercera función, propia a los nombres de autor en particular: clasificar. Aristóteles permite agrupar en un conjunto coherente y consistente, una serie de escritos, tales como la Poética, la Política, la Física o la Metafísica, entre otros, excluyendo El tratado de los colores de Teofrastro, o La retórica de Alejandro, atribuida a Anaxímenes de Lámpsaco. El nombre de autor es concomitante a la noción de obra.

El hecho de que un mismo individuo, llamado Jorge Mario Varlotta Levrero, decida utilizar, para firmar sus obras, alternativamente, Mario Levrero o Jorge Varlotta, no deja de afectar, en mayor o menor medida, estas tres funciones. La dificultad para nombrar al autor en las entrevistas realizadas en los años 1970-1980 no es el producto de un mero desliz. Estos nombres propios tal vez se refieran a un mismo sujeto, pero asociándolo a descripciones diferentes, mezclando el Todo y la Parte, el Uno y la Fracción, dificultando, por momentos, la clasificación de sus escritos.

Mario Levrero no es el ortónimo del autor, en el sentido estricto del término, si por ortónimo hay que entender la coincidencia entre el nombre de autor y el nombre civil. El nombre civil de este individuo da lugar a dos nombres de autor. ¿Podría hablarse entonces de semi-ortónimos? Si la respuesta fuera afirmativa, ¿es posible que uno de estos semi-ortónimos (Mario Levrero) sea más ortónimo que el otro semi-ortónimo (Jorge Varlotta)? ¿Levrero sería el ortónimo principal y Varlotta el ortónimo secundario? 


\section{6-... sabía que Jorge Varlotta no podía escribir eso.}

21 literario es un objeto extraño, fabricado por una personalidad ajena al autor, aunque no completamente : la literatura viene directamente armada del inconsciente, en un proceso automático, por no decir mágico-espiritual, como el crecimiento de una planta. Mario Levrero reformula la "Explicación falsa de mis cuentos" de Felisberto Hernández. Basta con leer "Sobre los mecanismos de la creación".

Pero por cierto, no se trata aquí del inconsciente freudiano. El inconsciente freudiano es intersticial, intermitente, insistente. Se manifiesta con el retorno de lo reprimido, cuando la censura falla y la consciencia tambalea con un lapsus, un acto fallido, un síntoma y el lenguaje maquillado de los sueños. El inconsciente invocado por Jorge Mario Varlotta 
Levrero, en cambio, se presenta, de forma masiva y directa, como abolición de la conciencia por la irrupción de otra conciencia, tal como ocurre en el estado hipnótico, la posesión, el trance mediúmnico, o aquello que los parapsicólogos llaman prosopopesis.

Por este término, inventado por René Sudre en su Traité de parapsychologie, a partir del griego prosopon, máscara de teatro o personaje, hay que entender un cambio brusco, espontáneo o provocado, de personalidad. En el Manual de parapsicología, Levrero escribe, a propósito de este fenómeno: "en determinadas circunstancias, ciertos sujetos abandonan su personalidad habitual para asumir otra completamente distinta. Sufren una distorsión de la memoria, y una transformación de los rasgos de carácter. Pueden tener nombre, ideas y tendencias diferentes. La nueva personalidad puede conocer a la anterior, pero hablar de ella como si fuera un extraño o un enemigo"10.

¿Mario Levrero es una personalidad diferente de Jorge Varlotta? ¿Mario Levrero es el inconsciente de Jorge Varlotta, que irrumpe y lo remplaza en determinadas ocasiones?

\section{8- Personalidades múltiples}

31 Aquello que está fundado en un sentimiento de unidad, puede estallar y fragmentarse. El sujeto puede olvidar quién es y adoptar otros nombres, otro pasado y otra vida. Para designar esta experiencia de disgregación psíquica, la ciencia decimonónica inventó un nombre : personalidades múltiples.

Sea el caso de Clara Norton Fowler, de Boston, conocida por la literatura médica como Miss Beauchamp, tratada por el Dr. Morton Prince. Esta paciente presentaba cuatro personalidades diferentes, que alternaban no sin conflicto: Miss Beauchamp 1 (personalidad neurasténica, inhibida, torturada, devota, depresiva), Miss Beauchamp 2 (personalidad que aparece bajo hipnosis, que conoce a Miss Beauchamp 1, pero que Miss Beauchamp 1 desconoce), Miss Beauchamp 3 (personalidad jovial, despreocupada, enemiga de la religión, conocida con el nombre de Sally y le hace la vida imposible a Miss Beauchamp 1, enviándole cartas con insultos o dejándole en la mesa de luz caja con alimañas) y Miss Beaucamp 4 (personalidad calma, pero desmemoriada, que desconoce quién es el Dr. Prince, a quien confunde con cierto Mr. Jones, su preceptor, fijada a un traumatismo acaecido varios años antes). ¿Cuál es la verdadera Miss Beauchamp? se pregunta perplejo Morton Prince en The Dissociation of a Personality ante esta proliferación de personalidades. Al final del tratamiento, parece encontrar una repuesta, al reunir a Miss Beauchamp 1 y Miss Beauchamp IV en una única personalidad, encerrando a la odiosa Miss Beauchamp 3, alias Sally, en la bastilla mental de la cual se había evadido.

Hay aquí, no un psiquismo unitario (el Yo) o dual (consciente/inconsciente), sino plural (personalidad 1, 2, 3, 4). Las personalidades que irrumpen en estos estados de fragmentación no forman parte de la personalidad principal, por cierto, pero a la vez tampoco le resultan completamente ajenas. De hecho, esta multiplicación de personalidades termina borrando la diferencia entre lo familiar y lo extraño, el Yo y el no-Yo. Entre las diferentes personalidades, hay un aire de familia: se conocen o se desconocen, se dominan o someten, una es la negación o la inversión de la otra. Se puede obtener una personalidad por sustracción de los atributos de otra. De ahí que de las sumas de las diversas personalidades, no resulte un todo. 


\section{9- Zona de intersección}

A decir verdad, la producción de Jorge Mario Varlotta Levrero, lejos de repartirse en dos autores, con contornos claramente delimitados, uno llamado Mario Levrero y el otro Jorge Varlotta, uno literario y otro no-literario, uno bajo tutela de la madre y el otro bajo la sombra del padre, uno representación de lo inconsciente e involuntario y el otro encarnación de lo consciente y voluntario, presenta zonas de conflicto e intersección, como por ejemplo, cuando el Nick Carter de Jorge Varlotta, al ser reeditado, termina firmado por Mario Levrero.

De esta operación, quedaron residuos no asimilables - por ahora- al nombre Mario Levrero. Destituido como autor, el nombre del otro semi-ortónimo no desaparece por completo. Entre septiembre de 1981 a enero de 1982, la revista Superhumor publica cinco episodios de Bill Carter, con la firma Jorge Varlotta, que, a pesar del cambio de nombre del protagonista, prolongan las aventuras de Nick Carter.

Este caso de substitución no es una excepción. En mayo de 1970, en el número 7 de $E l$ lagrimal trifurca, aparecen dos de los seis ejercicios de natación, que Levrero publica en Espacios libres, en 1987, atribuidos a Nat Persing, de quien puede leerse la siguiente biografía :

Nació en Austin (Texas) en 1940. En 1955 se traslada a Uruguay, donde reside actualmente. Es escritor bilingüe y traduce sus trabajos del inglés al castellano y viceversa. Los "ejercicios de natación", fueron escritos originariamente en castellano $^{11}$.

Mario Levrero, Jorge Varlotta, y los otros, se construyen en el intervalo, la yuxtaposición, la reconfiguración. Este conjunto de autores no forman un sistema coherente y consistente. La suma de Jorge Varlotta y Mario Levrero no da una totalidad, muy por el contrario, la desbaratan. Representan subjetividades que no se dejan pensar en términos de yo. Son más bien autores embrionarios, o si se prefiere, teratomas.

\section{0- Teratomas y psicotomas}

38 Un teratoma, le explica un genetista al narrador de "Precaución", que tiene un quiste sebáceo en el cráneo, "es una especie de hermano mellizo que no tuvo la fortuna de desarrollarse por completo y en forma independiente" ${ }^{12}$. De esta criatura abortada, queda un residuo que se manifiesta como quiste o tumor encapsulado con pelos, huesos o dientes. Este doble embrionario puede encarnarse en un psicotoma, es decir, "una formación psíquica que aparece como un cuerpo extraño a la psique, con cierto grado de autonomía, limitado en su desarrollo pero que vive a expensas del individuo que lo posee" ${ }^{13}$.

Como en el caso de las personalidades múltiples, entre el teratoma y su huésped hay una relación de ajenidad y familiaridad, autonomía y subordinación, que amenaza toda tentativa de unidad y totalidad. El narrador se pregunta, en determinado momento, si su quiste sebáceo no es un teratoma todo cerebro y el día en que resuelva operárselo se verá privado de sus ideas más brillantes. Este temor termina borrando las jerarquías. El que piensa, el que inventa, y el que escribe es el otro (pero un otro abortado, residual). 

un psicotoma de Levrero?

\section{1- Pessoa : heterónimos, semi-heterónimos y sub- heterónimos}

41 Otro autor que ha explotado las posibilidades de la desintegración de la personalidad para inventar una multitud de autores es, por cierto, Fernando Antonio Nogueira Pessoa, que, en 1928, en una nota bibliográfica publicada en la revista Presença, declara :

Lo que Fernando Pessoa escribe pertenece a dos categorías de obras a las que podemos llamar ortónimas y heterónimas. No se podrá decir que son autónimas o pseudónimas, porque en verdad no lo son. La obra pseudónima es del autor en su persona, salvo en el nombre que firma; la heterónima es del autor fuera de su persona, es de una individualidad completa y fabricada por él como lo serían los decires de cualquier personaje de cualquier drama suyo. Las obras heterónimas de Fernando Pessoa están hechas por, hasta ahora, tres nombres de persona : Alberto Caeiro, Ricardo Reis, Alvaro de Campos. Estas individualidades deben ser consideradas como distintas del autor ${ }^{14}$.

Al margen del trío de heterónimos oficialmente reconocidos como tales, autores distintos del autor, existen una nebulosa de semi-autores y sub-autores, inventados por Fernando Pessoa en borradores que fue atesorando en un baúl, que no han gozado de la misma visibilidad y quedaron fuera de la famosa coterie.

Tenemos, desde luego, a Bernardo Soares, un oscuro contador que trabajó toda su vida en una oficina de Lisboa, autor de El libro del desasosiego, reducido al estatuto de semiheterónimo. "Es un semi-heterónimo", explica Pessoa, en 1938, en su carta a Adolfo Casais Monteiro, "porque, sin ser mi personalidad, es, no distinta de la mía, sino una simple mutilación de ella. Soy yo menos el raciocinio y la afectividad".

Otro semi-heterónimo, menos conocido que Soares, es el barón de Teives, autor de un único manuscrito, intitulado La educación del estoico. En algunos papeles, Pessoa explica que los semi-heterónimos son personalidades "míamente ajenas", que escriben con su mismo estilo y gramática, pero sin ser él.

Si los heterónimos son autores enteros y autónomos, fabricados por adición de una personalidad a la personalidad del autor, los semi-heterónimos son autores parciales, obtenidos por una mutilación de la persona del autor, o, si se prefiere, por una sustracción de atributos.

Además de heterónimos y semi-heterónimos, Pessoa inventó una legión de subheterónimos, es decir, personalidades literarias apenas esbozadas en papeles, pero que no llegaron a cuajar como individualidades, entre los cuales figuran Alexander Search (gemelo de Pessoa, puesto que nació en Lisboa el mismo día, escribió en inglés varias composiciones poéticas, como así también un pacto con Jacob Satán), su hermano Charles Search (también nació en Lisboa, pero dos años antes que Alexander, traductor), Charles Anton, (megalómano, dipsómano, degenerado superior, poeta y humorista, esbozó una teoría de la percepción y un ensayo de literatura policial, entre sus poemas sobresalen el epitafio de Dios), Thomas Cross (traductor al inglés de los poemas de Alberto Caeiro y vulgarizador también en inglés de la cultura y poesía portuguesa), su hermano I.I. Cross (un crítico literario, particularmente interesado por la modernidad, que dejó algunas notas en inglés sobre Alberto Caeiro y Alvaro de Campos), su otro hermano A.A. Cross 
(autor de acertijos y crucigramas), Jean Seul de Méluré (poeta y ensayista de lengua francesa, dejó algunos papeles satíricos, intitulados "Des Cas d'Exhibitionnisme", "La France en 1950" y "Messieurs les Souteneurs"), António Mora (discípulo de Caeiro, filósofo, fundador del neopaganismo, autor de El regreso de los dioses), Raphael Baldaya (filósofo, astrólogo, ocultista, del cual se conocen algunos fragmentos de un tratado de la negación y unos principios de metafísica esotérica), Carlos Otto (autor de un sueño de Georgias y de un epigrama sobre el origen de las religiones, dejó también un borrador de un Tratado de lucha libre), Frederico Reis (autor de un solo texto crítico sobre el epicureísmo triste de la poesía de su primo Ricardo Reis), Pêro Botelho (autor un libro de cuentos, del cual solo se conoce "El Vendedor del tiempo")... y así sucesivamente.

Esta lista, por cierto incompleta, tendría que demostrar que, entre el ortónimo y los heterónimos, el yo y el no-yo, existen los más variados estados intermedios de yoes desmigajados.

La figura del autor es concomitante de la forma que adoptan sus escritos. Si a los heterónimos corresponden una obra con un estilo y un contorno más o menos definidos, a estos semi-heterónimos y sub-heterónimos les corresponden borradores, es decir, textos fragmentarios, inacabados y abortados, que por su precariedad escaparon a la publicación. Si el heterónimo es definido como autor ajeno a la persona del autor, los subheterónimos son más bien autores larvarios, que no se dejan pensar en términos de individualidad. Su existencia se reduce, en muchos casos, a un nombre y a unas notas garabateadas en un papel.

Los sub-heterónimos revelan, entre otras cosas, otra función de los nombres de autor : la función performativa. Para hacer existir un autor imaginario, basta con inventarle un nombre.

\section{2- Más nombres y autores}

50 Paralelamente a la utilización de sus dos semi-ortónimos, Jorge Mario Varlotta Levrero utilizó otros nombres para firmar textos humorísticos y periodísticos, publicados en diferentes revistas rioplatenses, de vida más o menos efímera, como Alvar Tot, Tía Encarnación, Bartleby Lavalleja, Ange de la Branche, Edipus Leroi, Profesor Hybris, Profesor Vrlachki, Profesor Off, Sofanor Rigby, Jalbert Klutsh, Carlos Nicole, J. Varli, y muchos otros.

51 Estos nombres no tienen la misma importancia. Algunos son empleados una sola vez, como es el caso de Edipus Leroi o de Ange de la Branche, que nacen y mueren en un número de la revista uruguaya Misia Dura; o el profesor Hybris, que firma un solo texto, en la revista uruguaya Privada, en los años ochenta. Tanto Edipus Leroi, como Ange de la Branche y el profesor Hybris son vulgarizadores burlescos del psicoanálisis, la parapsicología o la crítica literaria.

52 En este caso, tal vez sea posible hablar de pseudónimos. Pero, dado que en este autor no hay centro sino dos focos, o si se prefiere, no hay un ortónimo sino dos semi-ortónimos, ¿se trataría de pseudónimos de quién? ¿De Mario Levrero ? ¿De Jorge Varlotta ? ¿De Jorge Mario Varlotta Levrero?

Otros, como Alvar Tot, Tía Encarnación o Bartleby Lavalleja o el profesor Vrlaschki, tienen una vida más longeva. Son autores dobles : publican los mismos textos en ambas orillas del río de la Plata, con dos décadas de diferencia. Nacen en Misia Dura y sobreviven 
más allá de los años 1970, hasta fines de los años 1980, en publicaciones uruguayas, como Privada, El Dedo, o Guambia, o argentinas, como Superhumor. A diferencia de Edipus Leroi, Ange de la Branche y el profesor Hybris, su producción es más copiosa y constituye una serie.

Alvar Tot (de hecho, un anagrama de Varlotta) se dedicó a inventar crucigramas de vanguardia, y en ciertas oportunidades, tomó la pluma para reflexionar sobre el humor, como en el artículo publicado, en 1983, en el № 9 de Guambia, ¿Y usted... de qué se ríe?

El profesor Vrlachki (de hecho, ¿una corrupción de Varlotta?), como el profesor Hybris o Edipus Leroi, se dedica a la vulgarización de la ciencia, en su caso, la geometría y dedica sus Lecciones de geometría, al punto y a la recta.

Tía Encarnación es un avatar femenino, que se especializa en los consejos sentimentales, hogareños o estéticos, mezclando estereotipos de la prensa femenina y absurdo.

Bartleby Lavalleja es autor de algunas biografía de insignes hombres de ciencia, como Jabert Klutch, inventor del choclo; Alterio, inventor de la memoria; Wellington Labronca, alias bronquita, metafísico del arrabal; Isidore Ducasse, también conocido como el conde de Lautréamont, inventor en Montevideo de los corasanes, que los franceses llamaron croissants; Torndyke, un deshollinador inglés que descubre una extraña ley de casualidad, que pone en relación todo con todo, sobre todo aquello que no tiene ninguna relación ; o Giambattista Grozzo, autor de Pierre Ménard, autor del Quijote. Esta última biografía revela, tal vez de manera demasiado transparente, la impronta borgesiana del modelo bartleby-lavallejiano.

En estos casos, no se trata de utilizar, como en la simple pseudonimia, un nombre imaginario para firmar un texto. Si bien Mario Levrero se niega a considerarlos como heterónimos, estos autores larvarios, que no llegaron a construir una obra autónoma, escribieron textos diferentes de los que solía escribir y firmar Mario Levrero. En una entrevista con Elvio Gandolfo, podemos leer :

Fue una época muy divertida. Por otra parte, fue la única vez que logré cobrar una especie de sueldo por lo que hago. Y la única vez que tuve que trabajar contra reloj, forzando la inspiración. A veces tenía que entregar un texto en 24 horas, y no se me ocurría absolutamente nada. Entonces aparecían textos completamente distintos de los que acostumbraba hacer : por ejemplo, la famosa ley de Thorndyke [sic], firmada como "Lavalleja Bartleby", descordó totalmente la intención, que inicialmente era llenar de cualquier manera un par de carillas. De paso, creo que esa Ley de Thorndyke [sic] es la misma "ley del sincronismo acausal" que mucho tiempo después encontré en Jung ${ }^{15}$.

\section{3- Literatura mayor, literatura menor}

Jorge Mario Varlotta Levrero se vale de estos nombres inventados para firmar textos, artículos y crucigramas, publicados principalmente en revistas satíricas o de entretenimiento. Los pseudónimos y sub-heterónimos, en principio, están asociados a la cultura de masa y al formato periódico, mucho más efímero y marginal, que el formato libresco, asociado a la Literatura. En este sentido, forman parte de la constelación Nick Carter. Y, al igual que Nick Carter, trabajan con materiales que provienen de la cultura de masa, no sin borrar la diferencia entre literatura mayor y literatura menor.

Nick Carter es más que un mero folletín policial. El título no solo se refiere a un personaje inventado en 1886, por John Caryell, para una dime novel, publicada en el New York Weekly, 
llamado precisamente Nick Carter, sino también a una conocida novela de Faulkner, As I lay dying, Mientras yo agonizo. El folletín mezcla estereotipos de la cultura popular con procedimientos propios de las vanguardias narrativas, como pueden ser la disolución de la identidad del narrador, desgarrado entre la primera, la segunda y la tercera persona o la proliferación de mises en abyme. Del mismo modo, Tía Encarnación mezcla los estereotipos de la prensa femenina con los encuentros fortuitos entre paraguas y tablas de disección, Edipus Leroy o el profesor Hybris vulgarizan y tergiversan saberes prestigiosos, como el psicoanálisis o la geometría, poniendo en relación ingenio y lugar común. Bartleby Lavalleja publica, con la misma desenvoltura, en revistas satíricas como Superhumor o en revistas literarias como El lagrimal trifulca, junto a Mario Levrero, Jorge Varlotta y el efímero Nat Persing.

Esta disociación onomástica humorística tiene una fuerte impronta paródica. A cada nombre inventado, le corresponde un blanco contra el cual disparar, que va del formato ortodoxo de los crucigramas a la literatura borgesiana, sin olvidar la gauchesca o los autores de lo que fue dado en llamar nueva narrativa latinoamericana. A Jorge Mario Varlotta Levrero le resulta más fácil asesinar a ciertas figuras tutelares y fundacionales, esto es, matar al padre, firmando con un nombre imaginario. Una vez más, la invención de nombres es una estrategia para contornear la prohibición y la transgresión, diciendo en un género menor, con el nombre y la personalidad de otro, aquello que no es posible decir en nombre propio, en un género mayor.

\section{4- La obra de Mario Levrero}

62 En esta nebulosa de nombres de autor, el semi-ortónimo Mario Levrero, por cierto, ocupa un lugar privilegiado, a tal punto que termina imponiéndose como ortónimo del autor, eclipsando a su hermano mellizo, como así también a los pseudónimos y subheterónimos. La hegemonía de este nombre está estrechamente asociada a la construcción de una obra, según una gradación que va de la desintegración onomástica a una tentativa de unificación y jerarquización en torno al nombre propio Mario Levrero.

63 Al principio de su carrera literaria, Jorge Mario Varlotta Levrero no hace más que multiplicar los nombres de autor. Es a fines de los años 1960 y principio de los años 1970, cuando son publicados en Misia Dura los textos firmados con los pseudónimos/subheterónimos de Tía Encarnación, Bartleby Lavalleja, J. Vrlachki, Edipus Leroi y compañía. Los pseudónimos/sub-heterónimos irrumpen en un momento bien preciso : al principio. En esta etapa inaugural, la utilización de nombres imaginarios, además de una función paródica y crítica, pudo haber desempeñado una función formativa. Es también al principio de su carrera cuando se produce un máximo de disociación entre Jorge Varlotta (que firma Nick Carter) y Mario Levrero (que firma La máquina de pensar en Gladys, La ciudad o El lugar).

64 En los años 1980, si bien Jorge Mario Varlotta Levrero vuelve a publicar los mismos textos firmados con nombres de autores imaginarios que publicó en años 1970 en revistas uruguayas y argentinas; si bien Jorge Varlotta publica Bill Carter en Superhumor y las historietas Los Profesionales o Santo Varón; Mario Levrero comienza a afirmarse como nombre de autor principal. Levrero publica no solamente Fauna/Desplazamiento o compilaciones de relatos, como Aguas salobres o Espacios libres, en De la Flor o Punto Sur Literatura, esto es, en editoriales más visibles, sino que también comienza a afianzar su 
figura en la esfera pública, gracias a las entrevistas realizadas por Gandolfo, Rafael Courtoisie, Eduardo Berti, Cristina Siscar, Carlos María Domínguez, entre otros, publicadas en revistas de cierta difusión, como Brecha en Uruguay o Crisis en Argentina.

A principios de los años 1990, se produce un punto de inflexión, marcado por dos hechos.

El primero es que Jorge Mario Varlotta Levrero deja de publicar textos firmados con pseudónimos/sub-heterónimos. El segundo es que, cuando en 1992, Nick Carter es reeditado por la editorial Arca, en Uruguay, Mario Levrero se apropia de este texto, en una edición que viene acompañada por una bibliografía establecida por Pablo Rocca, pero preparada por el autor, con la lista de textos publicados con semi-ortónimos, pseudónimos y sub-heterónimos, precedida de esta advertencia :

Ninguna de las operaciones precedentes se hubiera llevado a cabo sin la colaboración generosa y paciente de Jorge Mario Varlotta Levrero, quien suele firmar sus trabajos, convencionalmente literarios, con su nombre y apellido segundos, y casi todos los demás con los primeros. Dejo debida constancia de que entre esta disociación (no precisamente pseudonímica), se privilegiará el nombre "literario", esto es : no será aclarado en cada texto particular. Contrariamente, Jorge Varlotta y sus numerosos heterónimos revistan al final del asiento correspondiente entre paréntesis rectos en las ocasiones que corresponda su "autoría"16.

Con la autorización del autor, Pablo Rocca subordina toda la producción de Jorge Mario Varlotta Levrero al semi-ortónimo Mario Levrero, imponiendo en esta proliferación de nombres y autores imaginarios, un centro y una jerarquía. Ambos acontecimientos, apropiación de un texto firmado al principio por Jorge Varlotta y subordinación de todas las firmas al nombre de Mario Levrero, señalan la emergencia de una obra. O si se prefiere : la construcción de una obra, atribuida a Mario Levrero, es concomitante con ciertas operaciones onomásticas que aseguran la supremacía de este semi-ortónimo sobre los demás, reforzando las funciones de designación, atribución, apropiación, clasificación y totalización.

67 A partir de los años 1990, el espacio de los semi-ortónimos queda claramente definido. Mientras Jorge Varlotta se limita a firmar las reseñas bibliográficas aparecidas en El País, de Uruguay, en los años 1990 ; Mario Levrero expande su soberanía, firmando con su nombre las columnas publicadas en Postdata (que más tarde aparecen compiladas en Irrupciones I y II), publicando sin escrúpulos textos de literatura "menor" como Dejen todo en mis manos, o comenzando una etapa autobiográfica, que le dará una mayor visibilidad, en una convergencia de las figuras del autor, el narrador y el personaje, sin nunca llegar a ser Uno.

\section{NOTAS}

1. Mario Levrero, Un silencio menos. Conversaciones compiladas por Elvio Gandolfo, Buenos Aires: Mansalva, 2013, p. 15.

2. Ídem, p. 24.

3. Ídem, p. 27. 
4. Ídem, p. 27.

5. Ídem, p. 40.

6. Ídem, p. 72.

7. Un silencio menos, op. cit, p. 115.

8. Ídem, p. 55.

9. Ídem, p. 108.

10. Mario Levrero, Manual de parapsicología, Buenos Aires : Ediciones de la Urraca, 1979, pp. 20-21.

11. El lagrimal trifurca, $\mathrm{N}^{\circ} 7$, mayo de 1970, p. 57 [edición facsimilar, Buenos Aires: Biblioteca Nacional, 2015, p. 457].

12. Mario Levrero, « Precaución », El portero y el otro, Arca, Montevideo, 1992, p. 74.

13. Ibidem, p. 77.

14. Citado por Angel Crespo, La vida plural de Fernando Pessoa, Barcelona : Seix Barral, 1988, p. 158.

15. Mario Levrero, Un silencio menos, op. cit., p. 18.

16. Pablo Rocca, «Advertencia », en Mario Levrero, Nick Carter, Montevideo : Arca, 1992, p. 91.

\section{AUTOR}

\section{DIEGO VECCHIO}

Escritor, Université Paris 8 